\title{
Astenopia em docentes universitários durante a pandemia da COVID-19
}

\author{
Asthenopia in college lecturers during the COVID-19 pandemic
}

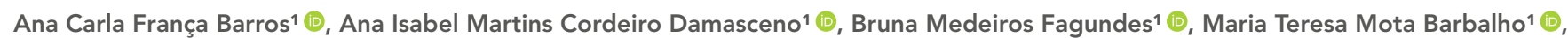
Matheus de Freitas d'Angelis' ${ }^{1}$ (]), Tarma Keullin Silva' ${ }^{10}$, Marcos Vinícius Macedo de Oliveira' ${ }^{1}$

${ }^{1}$ Centro Universitário FIPMoc, Montes Claros, MG, Brasil.

Como citar: universitários durante a pandemia da COVID-19. Rev Bras Oftalmol. 2022; 81:e0007.

universitários durante a pandemia da COVID-19. Rev Bras Oftalmol. 2022; 81:e0007.

https://doi.org/10.37039/1982.8551.20220007

\section{Descritores:}

Xeroftalmia; Diplopia; Educação à distância; Tempo de tela; Estresse ocupacional;

Manifestações oculares;

Docentes; COVID-19; Pandemia

Keywords: Xerophthalmia; Diplopia; Distance learning; Screen time; Occupational stress;

Eye manifestations; Faculty; COVID-19; Pandemics

Recebido: $13 / 7 / 2021$

Aceito:

29/11/2021

Autor correspondente: Marcos Vinícius Macedo de Oliveira Rua Carmino de Abreu, 240, apto. 101 Morada do Sol CEP: 39403-226 - Montes Claros, MG Brasil

E-mail: mvmoliv@gmail.com

Instituição de realização do trabalho: Centro Universitário FIPMoc, Montes Claros, MG, Brasil.

Fonte de auxílio à pesquisa: trabalho não financiado.

Conflitos de interesse: os autores declaram que não há conflitos de interesses.

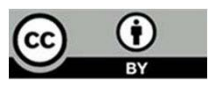

Copyright (C)2022

\section{RESUMO}

Objetivo: Avaliar sintomas astenópicos e fatores sociodemográficos, hábitos comportamentais e clínicos nos docentes universitários durante a pandemia da COVID-19.

Métodos: Trata-se de estudo transversal que avaliou a ocorrência de astenopia em 104 docentes. Questionários de sintomatologia visual validados foram adaptados para a coleta de dados. Houve comparação dos docentes quanto à ocorrência ou não de sintomas astenópicos, bem como foi aplicada regressão logística binária, para aferir a associação com variáveis independentes $(p<0,05)$.

Resultados: Houve maior aparecimento de sintomas astenópicos durante o período pandêmico, em que o tempo de exposição a telas parece ter sido o fator mais determinante. Além disso, os indivíduos com tempo de tela superior a 5 horas diárias, que faziam uso de telas para o lazer e usavam colírio/lubrificantes apresentaram significativamente maior chance de estar no grupo com sintomas astenópicos.

Conclusão: Foi identificada associação significativa entre a ocorrência de sintomas astenópicos e o uso de telas durante o período pandêmico, principalmente nos grupos com maior duração do tempo de tela. O estudo chama atenção para a saúde ocular de docentes universitários em ensino remoto, além de suscitar novos estudos para investigação desse quadro em distintos ambientes escolares.

\section{ABSTRACT}

Objective: To assess asthenopic symptoms and sociodemographic factors, behavioral and clinical aspects in college lecturers during the COVID-19 pandemic.

Methods: This is a cross-sectional study evaluating asthenopia in 104 lecturers. Some validated visual symptom questionnaires were adapted for data collection. Lecturers were compared regarding the occurrence or not of asthenopic symptoms, and binary logistic regression was applied to measure the association with independent variables $(p<0.05)$

Results: Asthenopic symptoms occurred more often during the pandemic, when exposure to screens was a determinant factor. The individuals with screen time longer than five hours a day, who used screens for leisure, and who used eye drops/lubricants were significantly more likely to be in the group with asthenopic symptoms.

Conclusion: A significant association was identified in occurrence of asthenopic symptoms and screen use during the pandemic period, especially in groups with longer screen time. The study draws attention to the eye health of college lecturers in distance learning, and the need for further research on this situation in different school environments. 


\section{INTRODUÇÃO}

A pandemia da doença pelo coronavírus 2019 (COVID-19) revela um impacto na população do mundo inteiro, com consequências assoladoras a curto prazo e ainda sem compreensão dos efeitos a médio e longo prazo. Com a disseminação do coronavírus da síndrome respiratória aguda grave 2 (SARS-CoV-2), veio a necessidade do distanciamento social, para evitar a propagação do vírus, e, como consequência, houve uma mudança na vida profissional e social, com aumento do uso de programas de videoconferência e instalação do ensino remoto emergencial, a partir do uso de computadores, celulares e tablet..$^{(1,2)}$

A população brasileira apresenta prevalência significativa de sintomas astenópicos associados ao uso de telas junto a cargas elevadas de trabalho, sendo 55\% provocados pelo uso de computadores, 73\% referiram fadiga ocular, 55\% descreveram queimação, 44\% relataram lacrimejamento e diminuição da acuidade visual, além da ser mais frequente no sexo feminino. ${ }^{(3)}$ Tais sintomas astenópicos foram descritos pela primeira vez em 1982 devido à contração excessiva e prolongada dos músculos ciliares, sendo, atualmente, fortemente relacionados à utilização prolongada de telas. ${ }^{\left({ }^{4}\right)}$

A implantação do ensino remoto emergencial como alternativa para a ministração de disciplinas teóricas e teórico-práticas configurou uma nova adaptação aos docentes universitários diante da pandemia, com o uso de novas plataformas virtuais de aprendizagem e, consequentemente, maior exposição a telas. Assim, novas demandas foram exigidas aos professores, tornando-os mais suscetíveis às consequências do uso excessivo de tais meios de comunicação, como o surgimento de sintomas astenópicos. ${ }^{(5)}$ A necessidade do uso de tecnologias no ambiente de trabalho, sobretudo no cenário de ensino remoto emergencial, demanda do educador mudança nas ferramentas utilizadas no processo ensino-aprendizagem e no ambiente de trabalho. ${ }^{(6,7)}$

O presente estudo objetivou avaliar a ocorrência de sintomas astenópicos e fatores associados em docentes do ensino superior durante a pandemia da COVID-19.

\section{MÉTODOS}

Trata-se de estudo transversal, retrospectivo e quantitativo, que avaliou a ocorrência dos sintomas astenópicos e sua relação com fatores sociodemográficos, hábitos comportamentais e clínicos em docentes universitários da cidade de Montes Claros (MG) em tempos de ensino remoto emergencial.

A amostra foi constituída de 104 docentes, selecionados por conveniência, entre uma população de 293 docentes de um centro universitário. A coleta de dados ocorreu nos meses de abril e maio de 2021, de forma remota, com contato via e-mail.

Os docentes foram submetidos a um formulário on-line contendo variáveis sociodemográficas, sendo elas: sexo (feminino ou masculino), idade, cor da pele (branca ou não branca), estado civil (com companheiro ou sem companheiro) e área de atuação (ciências exatas, ciências humanas ou ciências biológicas).

As informações sobre sintomatologia visual, saúde ocular, caracterização e tempo de uso de telas foram obtidas criando-se variáveis a partir de formulários validados: Visual Function Questionnaire (VFQ-25) do National Eye Institute (NEI) e Convergence Insufficiency Symptom Survey (CISS) desenvolvido pelo Convergence Insufficiency Treatment Trial (CITT). ${ }^{(8,9)}$ Nove informações foram coletadas: tempo de uso de telas (até 5 horas ou acima de 5 horas); tamanho da tela mais utilizada (smartphone/tablet ou acima de 14 "); distância média entre a cabeça e a tela mais utilizada (até $40 \mathrm{~cm}$ ou acima de $40 \mathrm{~cm}$ ); luminosidade do ambiente (mal iluminado ou bem iluminado); uso de telas para lazer (sim ou não); presença de doença oftalmológica (sim ou não); uso de óculos (sim ou não); uso de lentes de contato (sim ou não); e uso de medicamentos para aliviar os sintomas (não, colírio/lubrificante ou analgésico/anti-inflamatório não esteroide - AINE - ou relaxante muscular).

Além disso, para obter informações sobre a implementação do ensino remoto emergencial, foi indagado se existiu experiência prévia com aula on-line antes da pandemia (sim ou não) e a carga horaria diária de aulas ministradas on-line atualmente ( $<3$ horas ou $>3$ horas).

Tais variáveis foram cruzadas com a análise de sintomas astenópicos. A fim de definir a presença ou não de sintomas astenópicos, o questionário Quality of Life (QOL-COVD), desenvolvido pelo College of Optometrists in Vision Development, foi adaptado para este estudo, sendo composto de questões que estimam a presença e a frequência de sintomas associados às práticas visuais e podem influenciar na capacidade visual. ${ }^{(10,11)}$ Toda pergunta apresentava quatro opções de respostas sobre as frequências com que cada sintoma aconteceu, com base em escala Likert, pontuada de zero a três, sendo zero correspondente a "nunca", um a "às vezes", dois a "quase sempre" e três a "sempre". Foram utilizadas 12 perguntas que avaliavam a frequência de lacrimejamento (1), ardor (2), dor (3), coceira (4), ressecamento nos olhos (5), fotofobia (6), cefaleia (7), cansaço ou sonolência (8), olhos cansados (9), visão dupla (10), visão embaçada ou tela desfocada (11), além de dor nas costas ou pescoço (12). A pontuação desses itens foi somada para chegar a um escore final. A presença de sintomas astenópicos foi definida a partir do 
cálculo da mediana, sendo ela o ponto de corte da presença ou ausência de sintomas astenópicos. No estudo, a mediana dos escores foi nove; dessa forma, valores acima de nove corresponderam à classificação como presença de sintomas, sinalizando a hipótese de alteração visual, revelando a necessidade de uma avaliação especializada.

Os dados foram tabulados no programa Statistical Package for the Social Sciences (SPSS), versão 22.0. Inicialmente, foram realizadas estatísticas descritivas, para examinar as características sociodemográficas, comportamentais e clínicas da amostra. Quando apropriado, os resultados foram expressos em números absolutos (n), porcentagens (\%), médias e desvios-padrão (DP). Para testar a associação entre as variáveis independentes e a ocorrência de sintomas astenópicos, foi realizada análise multivariada por regressão logística binária. Nessa análise, docentes sem sintomas astenópicos atuaram como grupo de referência para todas as comparações. O modelo final foi obtido seguindo a técnica backward conditional para construir um modelo estatístico de variáveis que pudesse resultar em uma melhor explicação para a chance de apresentar sintomas astenópicos. Dessa forma, razões de chance $(\mathrm{RC})$ para queixas associadas à ocorrência de sintomas astenópicos e seus respectivos intervalos de confiança foram estimados com intervalo de confiança acima de 95\% (p <0,05).

Todos os participantes da pesquisa foram esclarecidos sobre objetivos do estudo e assinaram o Termo de Consentimento Livre e Esclarecido (TCLE), previamente aprovado pelo Comitê de Ética (parecer: 4.635.122/2021; CAAE: 44870921.1.0000.5109).

\section{RESULTADOS}

Os participantes da pesquisa estavam distribuídos nas seguintes áreas de conhecimento: 18 (17,3\%) participantes da área de ciências exatas, 34 (32,7\%) em ciências humanas e 52 (50\%) em ciências biológicas. Do total, 53 (51\%) eram do sexo masculino. A média de idade dos docentes foi de $42,3 \pm 8,2$ anos, variando de 29 a 60 anos. A maioria dos docentes vivia com companheiro (81,7\%). A distribuição de cor da pele foi homogênea: 54 (51,9\%) responderam cor branca e $50(48,1 \%)$ não branca.

A ocorrência de sintomas astenópicos foi identificada em 53,8\% dos docentes. Os principais sintomas astenópicos relatados ao menos uma vez pelos participantes da pesquisa foram cefaleia $(63,5 \%)$, olhos cansados $(61,5 \%)$, prurido ocular $(58,7 \%)$, ardor ocular $(58,7 \%)$, visão embaçada $(51,9 \%)$, fotofobia $(47,1 \%)$ e ressecamento ocular (42,3\%). Além disso, a maioria dos docentes descreveu sentir sintomas associados como cansaço/sonolência (67,3\%) e dor nas costas e pescoço $(52,9 \%)$.
A tabela 1 representa a distribuição de frequências descrevendo os participantes com e sem sintomas astenópicos em relação às variáveis da pesquisa. Houve maior aparecimento de sintomas astenópicos no período pandêmico, representando $62,5 \%$. O tempo de exposição a telas pareceu ser

Tabela 1. Ocorrência de sintomas astenópicos em relação às variáveis da pesquisa

\begin{tabular}{|c|c|c|}
\hline \multirow{2}{*}{ Variáveis } & \multicolumn{2}{|c|}{ Sintomas astenópicos } \\
\hline & Ausentes & Presentes \\
\hline \multicolumn{3}{|l|}{ Sexo } \\
\hline Feminino & $24(47,1)$ & $27(52,9)$ \\
\hline Masculino & $32(60,4)$ & $21(39,6)$ \\
\hline Idade, anos & $43,2 \pm 8,4$ & $42,9 \pm 8,0$ \\
\hline \multicolumn{3}{|l|}{ Estado civil } \\
\hline Com companheiro & $50(58,8)$ & $35(41,2)$ \\
\hline Sem companheiro & $6(31,6)$ & $13(68,4)$ \\
\hline \multicolumn{3}{|l|}{ Cor da pele } \\
\hline Branca & $29(53,7)$ & $25(46,3)$ \\
\hline Não branca & $27(54,0)$ & $23(46,0)$ \\
\hline \multicolumn{3}{|l|}{ Área do conhecimento } \\
\hline Ciências exatas & $12(66,7)$ & $6(33,3)$ \\
\hline Ciências humanas & $13(38,2)$ & $21(61,8)$ \\
\hline Ciências biológicas & $31(59,6)$ & $21(40,4)$ \\
\hline \multicolumn{3}{|l|}{ Tempo diário de uso de telas, horas } \\
\hline Até 5 & $18(75,0)$ & $6(25,0)$ \\
\hline Mais de 5 & $38(47,5)$ & $42(52,5)$ \\
\hline \multicolumn{3}{|l|}{ Tamanho da tela } \\
\hline Smartphone/tablet & $12(52,2)$ & $11(47,8)$ \\
\hline Acima de 14 polegadas & $44(54,3)$ & $37(45,7)$ \\
\hline \multicolumn{3}{|l|}{ Distância da tela, $\mathrm{cm}$} \\
\hline Até 40 & $32(52,5)$ & $29(47,5)$ \\
\hline Mais de 40 & $24(55,8)$ & $19(44,2)$ \\
\hline \multicolumn{3}{|l|}{ Luminosidade do ambiente } \\
\hline Mal iluminado & $3(42,9)$ & $4(57,1)$ \\
\hline Bem iluminado & $53(54,6)$ & $44(45,4)$ \\
\hline \multicolumn{3}{|l|}{ Uso de telas para lazer } \\
\hline Sim & $44(50,0)$ & $44(50,0)$ \\
\hline Não & $12(75,0)$ & $4(25,0)$ \\
\hline \multicolumn{3}{|l|}{ Uso semanal de telas para lazer, dias } \\
\hline Até 3 & $9(47,4)$ & $10(52,6)$ \\
\hline Mais de 3 & $35(50,7)$ & $34(49,3)$ \\
\hline \multicolumn{3}{|l|}{ Uso diário de telas para lazer, horas } \\
\hline Menos de 2 & $22(64,7)$ & $12(35,3)$ \\
\hline Mais de 2 & $22(40,7)$ & $32(59,3)$ \\
\hline \multicolumn{3}{|l|}{ Experiência prévia on-line } \\
\hline Sim & $7(63,6)$ & $4(36,4)$ \\
\hline Não & $49(52,7)$ & $44(47,3)$ \\
\hline \multicolumn{3}{|c|}{ Carga horária diária de aulas on-line, horas } \\
\hline Até 2 & $22(73,3)$ & $8(26,7)$ \\
\hline Mais de 2 & $34(45,9)$ & $40(54,1)$ \\
\hline \multicolumn{3}{|l|}{ Presença de doença oftalmológica } \\
\hline Sim & $45(51,7)$ & $42(48,3)$ \\
\hline Não & $11(64,7)$ & $6(35,3)$ \\
\hline \multicolumn{3}{|l|}{ Uso de óculos } \\
\hline Sim & $38(50,7)$ & $37(49,3)$ \\
\hline Não & $18(62,1)$ & $11(37,9)$ \\
\hline \multicolumn{3}{|l|}{ Uso de lentes de contato } \\
\hline Sim & $9(45,0)$ & $11(55,0)$ \\
\hline Não & $47(56,0)$ & $37(44,0)$ \\
\hline Início sintomas astenópicos & & \\
\hline Há 1 ano & & $30(62,5)$ \\
\hline Há mais de 1 ano & & $18(37,5)$ \\
\hline Uso de medicamentos & & \\
\hline Não & $40(64,5)$ & $22(35,5)$ \\
\hline Colírio/lubrificante ocular & $5(29,4)$ & $12(70,6)$ \\
\hline Analgésico/AINE/relaxante muscular & $11(44,0)$ & $14(56,0)$ \\
\hline
\end{tabular}


o fator mais determinante para associação com astenopia. Uma maior frequência de docentes no grupo que se expôs a telas por mais de 5 horas por dia relatou apresentar sintomas astenópicos. Além disso, entre aqueles que usavam telas para lazer acima de 2 horas diárias, também houve significativamente maior presença dos sintomas (59,3\%); $\mathrm{p}=0,048)$.

A tabela 2 expõe os resultados da análise multivariada. Essa avaliação indicou que indivíduos com tempo de uso de telas acima de 5 horas (RC de 3,631), que faziam o uso de telas para lazer ( $\mathrm{RC}$ de 7,450) e utilizaram colírio/lubrificantes (em relação aos que não usaram medicações; RC de 4,847) apresentaram significativamente maior chance de estar no grupo com sintomas astenópicos $(\mathrm{p}<0,05)$.

Tabela 2. Modelo ajustado de regressão logística binária estatisticamente mais significativo de associações entre as variáveis investigadas e a presença de astenopia

\begin{tabular}{|c|c|c|c|}
\hline Variáveis & $\mathrm{RC}$ & IC95\% & $\begin{array}{l}\text { Valor } \\
\text { de } p\end{array}$ \\
\hline \multicolumn{4}{|l|}{ Área do conhecimento } \\
\hline Ciências biológicas & Referência & & 0,026 \\
\hline Ciências exatas & 0,390 & $0,111-1,368$ & 0,0141 \\
\hline Ciências humanas & 2,503 & $0,904-6,926$ & 0,077 \\
\hline \multicolumn{4}{|l|}{ Tempo de uso de telas, horas } \\
\hline Até 5 & Referência & & \\
\hline Mais de 5 & 3,631 & $1,127-11,694$ & 0,031 * \\
\hline \multicolumn{4}{|l|}{ Uso de telas para lazer } \\
\hline Não & Referência & & \\
\hline Sim & 7,450 & $1,768-31,401$ & $0,006^{*}$ \\
\hline \multicolumn{4}{|l|}{ Uso de medicamentos } \\
\hline Não & Referência & & 0,057 \\
\hline Colírio/lubrificantes & 4,847 & $1,271-18,489$ & 0,021 * \\
\hline $\begin{array}{l}\text { Analgésico/AINE/relaxante } \\
\text { muscular }\end{array}$ & 2,002 & $0,689-5,816$ & 0,202 \\
\hline
\end{tabular}

\section{DISCUSSÃO}

Com a instituição da pandemia da COVID-19 em março de 2020 no Brasil, a internet passou a ter ainda mais relevância no ensino, pois as aulas, antes ministradas de forma presencial, foram, em sua maioria ou totalidade, adaptadas ao meio digital. ${ }^{(22)}$ Os docentes tiveram papel fundamental nessa mudança, sendo afetados diretamente pelo impacto do uso de telas, já que as diversas horas-aula, antes presenciais, tornaram-se on-line gerando uma exposição muito maior às telas, sendo este um dos fatores de risco para astenopia. . $5,13,14)^{2}$

O presente estudo não identificou associação significativa nas variáveis sexo, idade, estado civil, cor da pele, área de atuação, pausa durante o uso de tela, tamanho de tela, distância média entre a cabeça e a tela mais utilizada, luminosidade do ambiente, utilizar telas para lazer após o trabalho, ministrar aula on-line antes da pandemia, doença oftalmológica, uso de óculos ou uso de lentes de contato. No entanto, relações estatísticas foram observadas entre a presença de sintomas astenópicos e o tempo de uso de telas. A ocorrência foi maior no grupo que usava telas por mais de 5 horas diárias, se comparado ao grupo que não possuía sintomas astenópicos, com razão de chance de 3,631.

Tais achados vão ao encontro das prevalências encontradas em pesquisa da Vision Watch. ${ }^{(15)} \mathrm{O}$ estudo foi realizado em adultos americanos, e mais de $80 \%$ dos pesquisados relataram usar aparelhos eletrônicos por mais de 2 horas diárias. As principais consequências se manifestaram por meio de sintomas astenópicos, como dor no pescoço e ombros $(35 \%)$, cefaleia $(27,7 \%)$, tensão ocular $(32,4 \%)$, visão turva $(27,9 \%)$ e olhos secos $(27,2 \%)$.

O uso de eletrônicos exige maior esforço da visão para perto, resultando em maior esforço do músculo ciliar responsável pela acomodação, que, além de ser prolongada, necessita de maior atenção visual. Consequentemente, há uma redução na quantidade de piscadas por minuto, aumentando também a evaporação do filme lacrimal, com redução do nível de umidificação e no tempo de ruptura lacrimal, surgindo os sintomas de visão embaçada, olho seco, vermelho, irritado e demais sintomas astenópicos. ${ }^{(16)}$

Além disso, este estudo identificou maior chance de ocorrência de sintomas astenópicos em docentes que também usam telas para lazer (RC de 7.450), especialmente se for por tempo prolongado. Além disso, com maior tempo de tela, tem-se, também, como consequência, um maior tempo sentado. Sugere-se que se esse tempo for superior a 3 horas ao dia, pode haver repercussões nos níveis de triglicérides, insulina e circunferência abdominal. ${ }^{(17)}$ Apesar de ainda não existirem estudos que determinem um tempo de corte limite para determinar o tempo sentado prejudicial à saúde, é preciso ponderar esse comportamento e aumentar o nível de atividade física, combatendo o sedentarismo e prevenindo a exposição aumentada de telas e astenopia. ${ }^{(18)}$

Houve associação entre uso de medicamentos para alívio sintomático e presença de sintomas astenópicos. A análise estatística identificou chance quase cinco vezes maior de o uso de colírios lubrificantes ocorrer entre os que apresentam sintomas astenópicos em relação aos que não usam medicamentos. O uso de colírios lubrificantes corrobora maior umectação ocular e melhor filme lacrimal, justificando o uso preventivo e sintomático, pois os colírios se mostram eficazes em reduzir os sintomas. ${ }^{(19)}$ Já o uso de analgésicos, AINEs e relaxantes musculares é pertinente nas queixas de cefaleia e dor cervical como sintomático específico de primeira linha, aliviando a percepção da dor 
e das tensões musculares adjuntas ao uso prolongado de telas, não agindo na gênese da astenopia. ${ }^{(20)}$

É interessante ressaltar que, neste estudo, 62,5\% dos sintomáticos apresentaram esses sintomas há menos de 1 ano, corroborando fortemente as relações entre o aumento do uso de telas e a presença de sintomas astenópicos. Devido ao caráter excepcional, ainda não existem, na literatura, instrumentos validados específicos para avaliação de astenopia em docentes universitários e uso de telas, além de serem escassos os estudos nessa área, dificultando a comparação dos resultados. Embora o caráter transversal da pesquisa dificulte o estabelecimento de causa e efeito, há importante apontamento de fragilidades no sistema educacional, em relação à astenopia em professores. Portanto, sugere-se que estudos longitudinais demonstrem mais detalhamento sobre os fatores de risco associados, permitindo estabelecimento de medidas interventivas para melhoria da qualidade de vida e condições de trabalho dos docentes.

Este estudo levantou questões a respeito do uso excessivo de telas e sintomas astenópicos em professores. A atuação do docente, a partir destes resultados, deve ser repensada pela Medicina do Trabalho. É possível que, após o período pandêmico, novas relações de trabalho sejam estabelecidas, favorecendo o teletrabalho e a exposição prolongada a telas. A discussão desse problema é importante para instigar o desenvolvimento de normativas e/ou orientações sobre o tempo de exposição, descanso e realização de atividade física para prevenir astenopia.

Dessa forma, é fundamental a adoção de medidas, a serem estabelecidas pelas instituições de ensino em parceria com oftalmologistas, a fim de melhorar a qualidade da saúde ocular de docentes universitários. Além disso, a realização de novos estudos, para identificar a prevalência de sintomas astenópicos em docentes de outras universidades e do ensino fundamental e médio, faz-se necessária perante a escassez de estudos acerca de tal quadro durante o ensino remoto na pandemia da COVID-19.

\section{CONCLUSÃO}

A prevalência de sintomas astenópicos em docentes universitários apresentou associação com o uso de telas no período pandêmico, principalmente naqueles que apresentaram maior tempo de exposição a telas e maior tempo de uso de telas para o lazer e que faziam uso de colírios ou lubrificantes oculares. A ocorrência de tais sintomas possivelmente está associada com a adoção do ensino remoto de caráter emergencial, uma vez que o isolamento social foi uma das medidas adotadas para a proteção contra o SARS-CoV-2.

\section{REFERÊNCIAS}

1. Appenzeller S, Menezes FH, Santos GG, Padilha RF, Graça HS, Bragança JF. Novos tempos, novos desafios: estratégias para equidade de acesso ao ensino remoto emergencial. Rev Bras Educ Méd. 2020;44(Supl 01).

2. Nash C. Report on digital literacy in academic meetings during the 2020 COVID-19 lockdown. Challenges. 2020;11(2):20.

3. Santos M, Almeida A, Oliveira T. Profissionais a exercer em Call Center: principais fatores de risco e riscos laborais, doenças profissionais associadas e medidas de proteção recomendadas. Rev Portuguesa Saúde Ocup. 2018;5:50-62.

4. Norman K, Floderus B, Hagman M, Toomingas A, Tornqvist EW. Musculoskeletal symptoms in relation to work exposures at call centre companies in Sweden. Work. 2008;30(2):201-14

5. Valente GS, Moraes EB, Sanchez MC, Souza DF, Pacheco MC. O ensino remoto frente às exigências do contexto de pandemia: Reflexões sobre à prática docente. Research Society and Development. 2020;9(9). http:// dx.doi.org/10.33448/rsd-v9i9.8153

6. Garcia MB, Oliveira MM, Plantier AP. Interatividade e mediação na prática de metodologia ativa: o uso da instrução por colegas e da tecnologia na educação médica. Rev Bras Educ Méd. 2019;43(1):87-96.

7. González-Menéndez E, López-González MJ, Menéndez SG, González GG, Bayona TA. Principales consecuencias para la salud derivadas del uso continuado de nuevos dispositivos electrónicos con PVD. Rev Esp Salud Publica. 2020;93:e201908062.

8. Nunes AF, Nunes AJ, Monteiro PM, Pato MA. Desempenho visual: validação do inventário de eficiência visual em estudantes. Rev Bras Oftalmol. 2015;74(2):92-8.

9. Scheiman M, Mitchell GL, Cotter S, Cooper J, Kulp M, Rouse M, Borsting E, London R, Wensveen J; Convergence Insufficiency Treatment Trial Study Group. A randomized clinical trial of treatments for convergence insufficiency in children. Arch Ophthalmol. 2005;123(1):14-24.

10. Mello PR, Roma Ade C, Moraes Júnior HV. [Analysis of the life quality of infectious and non-infectious patients with uveitis using the NEI-VFQ-25 questionnaire]. Arq Bras Oftalmol. 2008;71(6):847-54. Portuguese.

11. Nunes FM, Oliva HN, Oliveira GS, França DS. Asthenopic symptoms prevalence in undergraduate students. Rev Bras Oftalmol. 2018;77(6):334-7.

12. Pasini CG, Carvalho E, Almeida LH. A educação híbrida em tempos de pandemia: algumas considerações. Observatório Socioeconômico da COVID-19 (OSE). 2020 [citado 2020 Out 26]. Disponível em: https://www. osecovid19.cloud.ufsm.br/media/documents/2021/03/29/Textos_para_ Discussao_09_-_Educacao_Hibrida_em_Tempos_de_Pandemia.pdf

13. Hodges C, Moore S, Lockee B, Trust T, Bond A. The difference between emergency remote teaching and online learning. Educ Review. 2020;27(1);1-9.

14. Todos pela Educação. Nota Técnica. Ensino a distância na Educação Básica frente à pandemia da Covid-19. 2020.Disponível em: https://www. todospelaeducacao.org.br/_uploads/_posts/425.pdf

15. The Vision Council. The vision council shines light on protecting sight - and health - in a multi-screen era. 2019.Disponível em: https://www. thevisioncouncil.org/blog/vision-council-shines-light-protecting-sightand-health-multi-screen-era

16. Comerio RS, Saraiva PG, Martins PS, Rodrigues MZ, Saager SB, Saraiva FP. Asthenopia in bankers: identification and analysis of risk factors. Rev Bras Oftalmol. 2017;76(2):70-3.

17. Staiano AE, Harrington DM, Barreira TV, Katzmarzyk PT. Sitting time and cardiometabolic risk in US adults: associations by sex, race, socioeconomic status and activity level. Br J Sports Med. 2014;48(3):213-9.

18. Rocha BM, Goldbaum M, César CL, Stopa SR. Comportamento sedentário na cidade de São Paulo: ISA-Capital 2015. Rev Bras Epidemiol. 2019;22:e19005.

19. Neves LR, D'Araujo JJ. Estudo dos sintomas visuais na síndrome relacionada ao computador e efeitos dos colírios lubrificantes em funcionários do hospital universitário Bettina Ferro de Sousa. Rev Eletrôn Acervo Saúde. 2019;11(8):591-91.

20. Melhado EM, Brugugnolli ID, Salis GV, Buck C, Goulart LA, Sucena TA, et al. Protocol of treatment of headache in the emergency in a university hospital. Headache Med. 2017;8(2):43-7. 